\title{
Million Per Ejaculate Unit
}

National Cancer Institute

\section{Source}

National Cancer Institute. Million Per Ejaculate Unit. NCI Thesaurus. Code C130189.

A unit of measurement equal to 10 to the sixth power of entities per unit equal to one ejaculation. 